\title{
Bedeutung der Abkürzungen für die einzelnen Abschnitte.
}

I. Allgemeine Physik.

I. 1. Maass und Messen.

I. 1a. Allgemeine Laboratoriumseinrichtungen.

I. 2. Dichtigkeit.

I. 3. Molecularphysik.

I. 3a. Krystallographisches.

I. 4. Mechanik.

I. 5. Hydrodynamik.

I. 6 Aërodyuamik.

I. 7. Cohäsion und Adhäsion.

I. 7A. Elasticität und Festigkeit.

I. 7B. Capillarität.

I. 7C. Löslichkeit.

I. 7D. Diffusion.

I. 7E. Absorption.

II. A kustik.

II. 8. Physikalische Akustik.

II. 9. Physiologische Akustik.

III. Optik.

III. 10. Theorie des Lichtes.

III. 11. Fortpflanzung, Spiegelung und Brechung des Lichtes.

III. 12. Objective Farben, Spectrum, Absorption.

III. 13. Photometrie.

III. 14. Phosphorescenz und Fluorescenz.

III. 15. Interferenz, Polarisation, Doppelbrechung, Krystalloptik.

III. 15A. Circularpolarisation.

III. 15B. Krystalloptik.

III. 16. Chemische Wirkungen des Lichtes.

III. 17. Physiologische Optik.

III. 17A. Der dioptrische Apparat des Auges.

III. 17B. Physiologie der Retina.

III. 17C. Farbensinn.

III. 17D. Psychische Verarbeitung der Gesichts-Eindrücke.

III. 17E. Wirkungen des Lichtes auf Pflanzen und niedere Thiere. 
III. 18. Optische Apparate.

III. 18A. Spiegel und Spiegelinstrumente.

III. 18B. Refractionsinstrumente.
a) Fernrohr und Theile desselben.
b) Mikroskop und Theile desselben.
c) Spectroskop.
d) Photographische Apparate.

III. 18C. Verschiedene optische Instrumente.

IV. Wärmelehre.

IV. 19. Theorie der Wärme und calorische Maschinen.

Allgemeines.

A. Erster Hauptsatz.

B. Zweiter Hauptsatz.

C. Gastheorie.

D. Anwendungen der mechanischen Wärmetheorie.

IV. 20. Thermometrie und Ausdehnung.

IV. 21. Quellen der Wärme.

IV. 21A. Mechanische Quellen der Wärme.

IV. 21B. Chemische Quellen der Wärme, Verbrennung.

IV. 21C. Physiologische Quellen der Wärme.

IV. 22. Aenderung des Aggregatzustandes.

IV. 23. Specifische Wärme, Calorimetrie.

IV. 24. Verbreitung der Wärme.

IV. 24A. Wärmeleitung.

IV. 24B. Wärmestrahlung.

IV. 24C. Radiometer.

V. Elektricitätslehre.

V. 25. Allgemeine Theorie der Elektricität und des Magnetismus und Diëlektricität.

V. 26. Elektricitätserregung.

V. 27. Elektrostatik.

V. 28. Batterieentladung.

V. 29. Galvanische Ketten.

V. 30. Galvanische Messapparate.

V. 31. Theorie der Kette.

V. 32. Elektrochemie.

V. 33. Thermoelektricität.

V. 34. Elektrische Wärmeerzeugung.

V. 35. Elektrisches Licht.

V. 36. Magnetismus.

V. 37. Elektromagnetismus.

V. 38. Elektrodynamik, Induction.

V. 39. Elektrophysiologie.

V. 40. Anwendungen der Elektricität.

VI. Physik der Erde.

VI. 41. Astrophysik.

VI. $41 \mathrm{~A}$. Allgemeines. Theorie der Gestirnbewegungen.

Beobachtungen von Observatorien.

VI. 41 B. Die Planeten und ihre Trabanten. 
VI. $41 \mathrm{C}$. Die Fixsterne und Nebel.

VI. 41 D. Die Sonne.

VI. $41 \mathrm{E}$. Die Kometen.

VI. 41F. Die Sternschnuppen.

VI. $41 \mathrm{G}$. Meteorsteine.

VI. $41 \mathrm{H}$. Das Polar- und Zodiakallicht.

VI. 42. Meteorologie.

VI. 42 A. Allgemeines. Theorien. Kosmische Meteorologie; allgemeine Eigenschaften der Atmosphäre (Zu-

VI. 42 B. Apparate. sammensetzung etc.).

VI. $42 \mathrm{C}$. Meteorologische Optik.

VI. $42 \mathrm{D}$. Temperatur.

VI. $42 \mathrm{E}$. Luftdruck und Höhenmessungen.

VI. $42 \mathrm{~F}$. Winde.

VI. 42 G. Feuchtigkeit, Wolken und Nebel.

VI. $42 \mathrm{H}$. Atmosphärische Niederschläge.

VI. $42 \mathrm{~J}$. Allgemeine Beobachtungen (Klimatologie), Beobachtungen auf Reisen.

VI. 43. Erdmagnetismus.

VI. 44. Atmosphärische Elektricität (Gewitter etc.).

VI. 45. Physikalische Geographie.

VI. $45 \mathrm{~A}$. Physik der Erde.

1. Ortsbestimmungen, Pendelbeobachtungen, allgemeine Eigenschaften der Erde (Dicjte etc.)

2. Boden- und Erdtemperatur.

3. Vulkane.

4. Erdbeben.

5. Hebungen und Senkungen, Gebirge, Thalbildungen, Niveauveränderungen, besondere Verwitterungserscheinungen.

6. Theorien der Erdbildung.

VI. 45 B. Physik des Wassers.

1. Meere (Oceanographie).

2. Seen und Flüsse.

3. Quellen, Grundwasser.

4. Eis, Eiszeit, Gletscher, Glacialphysik.

VI. 46. Geographie und Reisen, in denen physikalische Beobachtungen sich vorfinden. 